\title{
Transversal Loading Sensor Based on Tunable Beat Frequency of a Dual-Wavelength Fiber Laser
}

\author{
Hongyan Fu, Student Member, IEEE, Xuewen Shu, Chengbo Mou, Lin Zhang, Sailing He, Senior Member, IEEE,
} and Ian Bennion, Member, IEEE

\begin{abstract}
Microwave signal generation by using the photonic beating from a phase-shift fiber Bragg grating (PS-FBG)-based dual-wavelength laser is proposed and experimentally demonstrated. The dual-wavelength laser is formed by a linear cavity, in which a PS-FBG is used as a dual-wavelength selective component. Transversal loading on the PS-FBG enhances the birefringence of the optical fiber and consequently makes the transmission peak of the PS-FBG splitting into two sharp transmission peaks of orthogonal polarizations. The wavelength spacing between the two transmission peaks increases with the transversal loading on the PS-FBG, thus making the polarization beating frequency increase. This property is exploited in a transversal loading sensor.
\end{abstract}

Index Terms-Beating, microwave generation, phase-shift fiber Bragg grating (PS-FBG), transversal loading.

\section{INTRODUCTION}

$\mathbf{F}$ IBER Bragg gratings (FBGs) have been studied intensively during the last two decades due to their wide variety of industry applications, such as components in fiber-optics communication systems and many kinds of fiber-optics sensors to monitor physical properties like strain, temperature, transversal loading, refractive index, and so on [1]-[3]. In recent years, photonic generation of the microwave signal has attracted much research attention since it is very useful in the promising radio-over-fiber systems [4]. Many approaches utilizing FBGs to implementing photonic generation of a microwave signal have been proposed, including utilizing the dual-phase-shift grating [5] and fiber Fabry-Pérot interferometer [6] formed by two FBGs to achieve a single-longitudinal-mode (SLM) dual-wavelength laser, which can beat to generate a microwave signal at a certain frequency. Two very narrow transmission peaks can ensure the SLM operation of the dual-wavelength lasing; however, in both methods, the structure of FBG is either

Manuscript received February 28, 2009; revised March 30, 2009. First published May 08, 2009; current version published June 26, 2009. This work was supported by the Chinese Scholarship Council.

$\mathrm{H}$. Fu is with the Centre for Optical and Electromagnetic Research, State Key Laboratory of Modern Optical Instrumentation of Zhejiang University, Zhejiang University, Hangzhou 310058, China, and also with the Photonics Research Group, Aston University, Birmingham, B4 7ET, U.K. (e-mail: fuhongyan@coer.zju.edu.cn).

X. Shu, C. Mou, L. Zhang, and I. Bennion are with the Photonics Research Group, Aston University, Birmingham, B4 7ET, U.K. (e-mail: x.shu@aston.ac.uk; mouc@aston.ac.uk; 1.zhang@aston.ac.uk; i.bennion@aston.ac.uk).

S. He is with the Centre for Optical and Electromagnetic Research, State Key Laboratory of Modern Optical Instrumentation of Zhejiang University, Zhejiang University, Hangzhou 310058, China (e-mail: sailing@zju.edu.cn).

Color versions of one or more of the figures in this letter are available online at http://ieeexplore.ieee.org.

Digital Object Identifier 10.1109/LPT.2009.2021484 very complicated to be fabricated or needs very careful design about the fabrication parameters. Furthermore, in these reported methods, the frequency of the beating-generated microwave signals can be hardly tuned due to the inherent characteristics of the FBG structures.

In this letter, photonic generation of a microwave signal by using the polarization beating from a phase-shift fiber Bragg grating (PS-FBG)-based dual-wavelength laser is proposed and experimentally demonstrated. Two sharp transmission peaks of orthogonal polarizations in the stopband of the PS-FBG can be introduced by applying the transversal loading on the PS-FBG, which can be utilized as the wavelength-selective element in the linear cavity of the dual-wavelength laser. Stable dual-wavelength lasing has been achieved in the experiment, and by the polarization beating between the two wavelengths, a microwave signal at certain frequency has been generated. The wavelength spacing between the two transmission peaks of the PS-FBG can be tuned by changing the transversal loading applied on the PS-FBG, and thus the frequency of the beating-generated microwave signal becomes tunable. On the other hand, this approach can also be applied to monitoring the transversal loading variation. The proposed method is simple and easy to implement, and has great potential in the application of both photonic generation of a microwave signal and fiber-optics transversal loading sensor.

\section{OPERATION PINCIPLE AND EXPERIEMENT SETUP}

When a part of fiber is under the pressure disturbance, due to the photoelastic effect, the effective index will be changed [7], [8]. The effective index change for $x$-axis and $y$-axis polarization can be expressed, respectively, as

$$
\begin{aligned}
\left(\Delta n_{\mathrm{eff}}\right)_{x}= & -\frac{n_{\mathrm{eff}}^{3}}{2 Y}\left\{\left(p_{11}-2 v p_{12}\right) \sigma_{x}\right. \\
& \left.+\left[(1-v) p_{12}-v p_{11}\right]\left[\sigma_{y}+\sigma_{z}\right]\right\}
\end{aligned}
$$

and

$$
\begin{aligned}
\left(\Delta n_{\mathrm{eff}}\right)_{y}= & -\frac{n_{\mathrm{eff}}^{3}}{2 Y}\left\{\left(p_{11}-2 v p_{12}\right) \sigma_{y}\right. \\
& \left.+\left[(1-v) p_{12}-v p_{11}\right]\left[\sigma_{x}+\sigma_{z}\right]\right\}
\end{aligned}
$$

where $Y$ is the Young's modulus of the optical fiber, $v$ is the Poisson's coefficient, $p_{11}$ and $p_{12}$ are the components of the photoelastic tensor, and $\sigma_{x}, \sigma_{y}$ and $\sigma_{z}$ are the stress components for the directions $x, y$, and $z$, respectively. For a typical germanosilicate fiber, when a transversal loading is applied on the fiber, the effective index changes for the $x$-axis and $y$-axis have different values.

The Bragg wavelength of an FBG is dependent on the mode effective index of the fiber core $n_{\text {eff }}$ as $\lambda_{\mathrm{Bragg}}=2 n_{\mathrm{eff}} \Lambda$, where $\Lambda$ is the period of the FBG. When a phase shift of $\pi$ is induced 


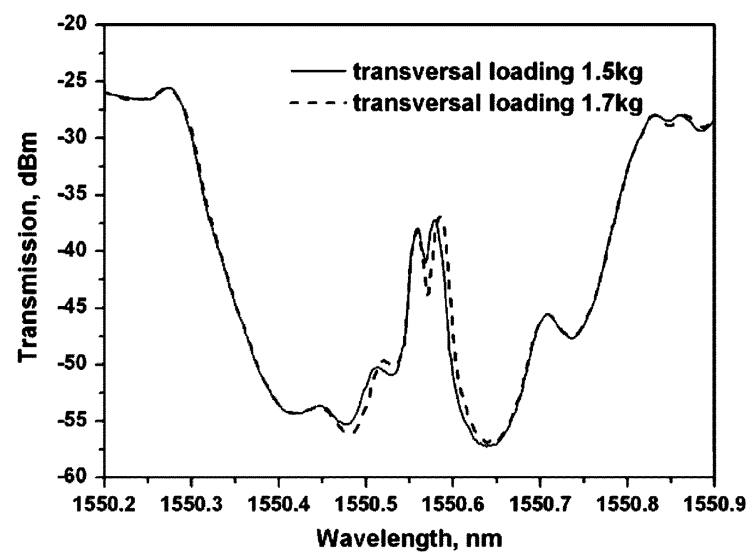

Fig. 1. Measured transmission spectra of the PS-FBG under different transversal load: $1.5 \mathrm{~kg}$ for solid line and $1.7 \mathrm{~kg}$ for dashed line.

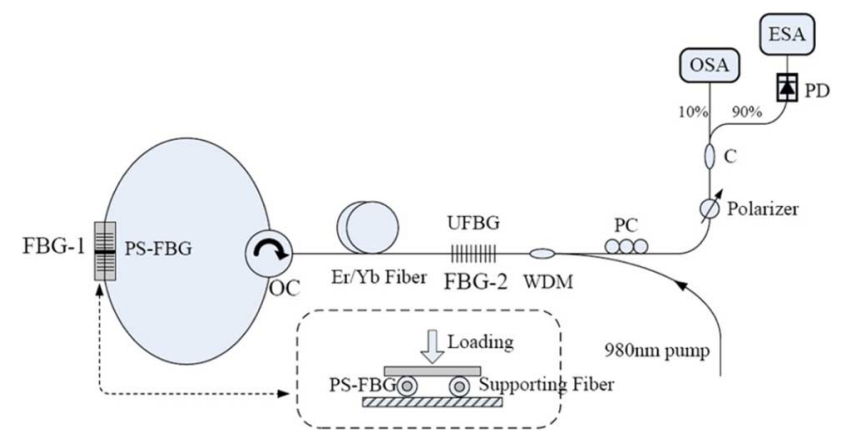

Fig. 2. Schematic diagram of the proposed dual-wavelength laser and the microwave generation; inset is the loading configuration.

in the FBG, there will be an ultranarrow transmission peak appearing in the stopband, and when the transversal loading is applied on the PS-FBG, due to the difference of the refractive index change of the fiber core, the transmission peak will split into two peaks. The wavelength spacing between the two peaks can change when the transversal loading varies. One example is shown in Fig. 1 (the solid line is for $1.5-\mathrm{kg}$ transversal load and the dashed line is for $1.7 \mathrm{~kg}$ ). We cannot see fully resolved sharp transmission peaks just because of the limited resolution of the optical spectrum analyzer $[(\mathrm{OSA}) \sim 0.01 \mathrm{~nm}]$.

The experimental setup of the PS-FBG-based dual-wavelength laser and photonic generation of the microwave signal is shown in Fig. 2. The cavity of the dual-wavelength laser is formed by FBG-1 (PS-FBG) with an optical circulator, a 1-m-long Er-Yb fiber, and FBG-2 [a uniform FBG (UFBG)]. FBG-1 is placed on the loading configuration shown in the inset (dashed block) in Fig. 2, and FBG-2 is mounted on a fiber stretcher. The Bragg wavelength of FBG-2 can be tuned by applying strain (i.e., by adjusting the fiber stretcher) to match with the two transmission peaks of the FBG-1. The laser is pumped by a $980-\mathrm{nm}$ laser diode coupled from a 980/1550-nm wavelength-division-multiplexing coupler spliced to the one end of the cavity to minimize the cavity length, and the laser output which passes through a polarization controller and a polarizer is launched into a 10:90 coupler (C). Ten percent of the laser output is monitored by the OSA (Ando AQ 6317), while $90 \%$ of the output goes into the photodetector [(PD) HP-11982A, $10 \mathrm{GHz}]$. The beating signal is observed by the electrical spectrum analyzer [(ESA) HP 70590A, $40 \mathrm{GHz}$.

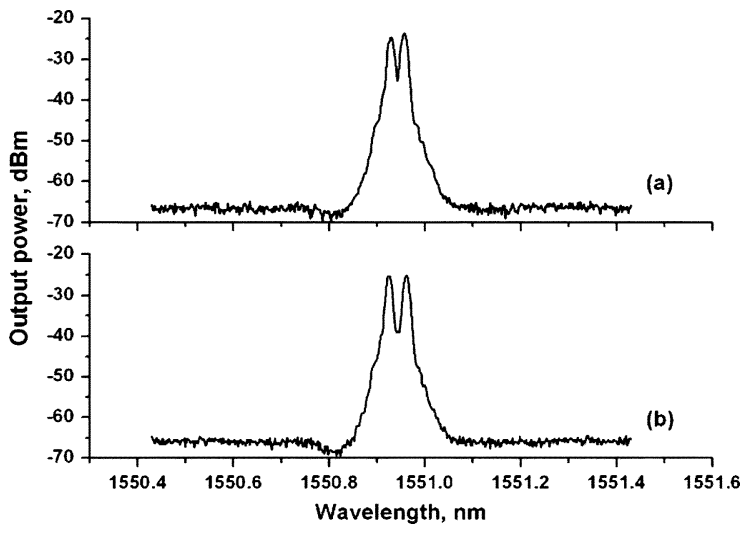

Fig. 3. Dual-wavelength laser output when (a) 5.0- and (b) 6.0-kg transversal loading are applied on the PS-FBG.

\section{EXPERIMENTAL RESULTS}

In the experiment, a PS-FBG with the reflection of $\sim 30 \mathrm{~dB}$ and transmission peak located around $1550.55 \mathrm{~nm}$ was used as FBG-1. A UFBG with the Bragg wavelength located around $1550.4 \mathrm{~nm}$ and the reflection of around $0.02 \mathrm{~nm}$ was employed as the FBG-2. The current of the 980-nm pump diode was set at $205.5 \mathrm{~mA}$ to achieve the lasing output. At first, no transversal loading was applied on the PS-FBG, and when we stretched the FBG-2 to match its Bragg wavelength with the unsplit transmission peak of the PS-FBG, a single wavelength lasing was achieved at $1550.55 \mathrm{~nm}$.

When the transversal loading was applied on the PS-FBG, dual-wavelength lasing was achieved since the transmission peak of PS-FBG split into two peaks induced by different index changes along the $x$-axis and $y$-axis of the fiber core. The polarization hole-burning in the cavity greatly enhance the dual-wavelength operation of the laser [9]. We then applied different transversal loads on the PS-FBG to change the wavelength spacing between the two polarization peaks. Fig. 3(a) and (b) shows the dual-wavelength laser outputs when 5.0- and 6.0-kg loads were applied on the PS-FBG in the experiment, respectively.

The stability measurement of the dual-wavelength laser was carried out for the 5.0-kg transversal load on the PS-FBG under room temperature. The laser output spectrum was measured every $5 \mathrm{~min}$ for a total period of $50 \mathrm{~min}$. The laser stability measurement result is shown in Fig. 4, from which one can see that the short-term stability of the dual-wavelength laser is good under room temperature in our experiment.

The beating-generated microwave signal can be observed by the ESA after optical beating on the PD. The microwave signals for 5.5- and $6.5-\mathrm{kg}$ transversal loads are shown in Fig. 5(a) and (b), respectively, from which one can see that the frequency of the beating microwave signal also increases when we increase the load.

The measured relationship between the value of transversal load and the frequency of the beating-generated microwave signal is depicted in Fig. 6, which shows a very good tuning linearity. From this tuning characteristic, we can expect that by tracking the frequency of the microwave signal, this scheme also has the potential to be applied as a transversal loading sensor. 


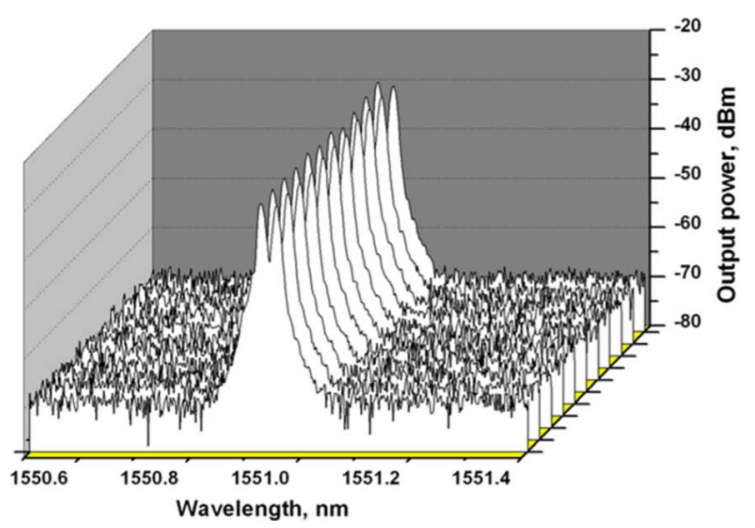

Fig. 4. Stability measurement result of the dual-wavelength laser output.

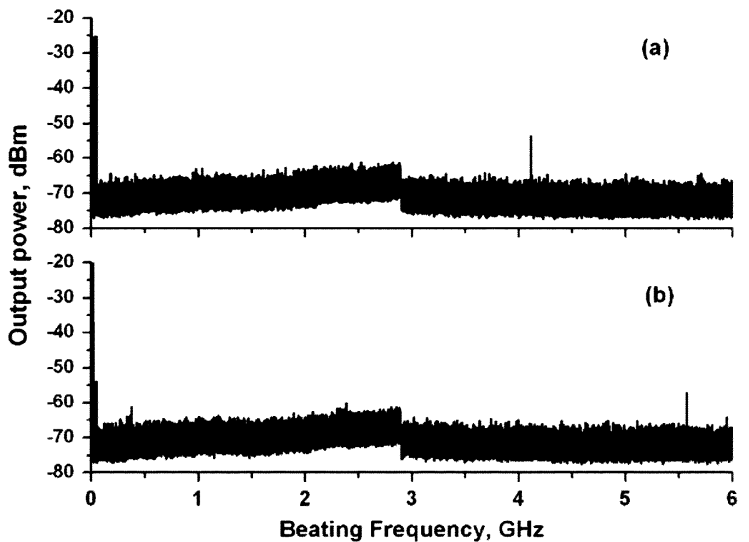

Fig. 5. Measured spectra of the beating microwave signal when (a) 5.5- and (b) $6.5-\mathrm{kg}$ transversal loading is applied.

\section{DISCUSSION AND CONCLUSION}

One may observe from Fig. 5 that there are several beating modes at the baseband, which means that the dual-wavelength laser does not strictly operate at the SLM operation state. This is because the longitudinal-mode frequency spacing of the laser cavity is slightly larger than the bandwidths of the transmission peaks of the PS-FBG. This problem can be solved by choosing another PS-FBG with higher reflection and narrower transmission bandwidth. Another issue is that the orientation of the applied transversal loading on the PS-FBG is an important factor to the beat frequency of the microwave signal. When the same value of loading is applied, the frequency of the beating-generated microwave signal may be different for the loading applied to the different orientation of the applied transversal loading, which means that this scheme may offer more tuning versatility. Furthermore, the beating frequency of the proposed scheme only relies on the wavelength spacing of two narrow transmission peaks of the PS-FBG with applied transversal load, and therefore, it is insensitive to the temperature.

In summary, a microwave signal generation utilizing optical beating of a PS-FBG-based dual-wavelength laser has been proposed. The PS-FBG behaves as a wavelength-selective element

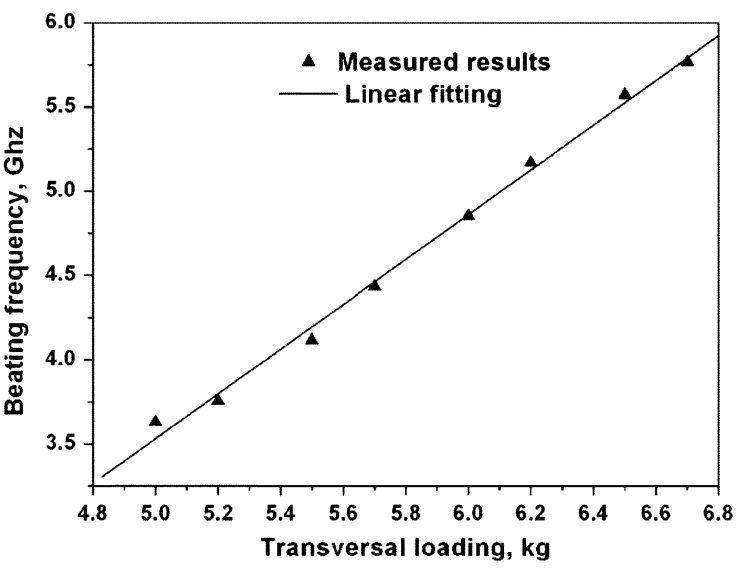

Fig. 6. Measured (triangles) and linear fitting (solid line) relationship between the frequency of beating microwave signal and the applied transversal loading.

in the laser cavity, whose transmission peak in the stopband can split into two sharp transmission peaks when transversal loading is applied on the PS-FBG. Stable dual-wavelength laser operation at room temperature has been achieved in the experiment. The wavelength spacing will change with the applied loading, and thus the beating frequency of the microwave signal is tunable. For the present method, the fabrication of the FBGs is easier and the scheme is simple to implement. Furthermore, the scheme can also be applied as a transversal loading sensor. Thus, the demonstrated PS-FBG-based dual-wavelength laser may have great potential for both next-generation communication systems and transversal loading sensing applications.

\section{REFERENCES}

[1] T. Erdogan, "Fiber grating spectra," J. Lightw. Technol., vol. 15, no. 8, pp. 1277-1294, Aug. 1997.

[2] Y.-J. Rao, "In-fibre Bragg grating sensors," Meas. Sci. Technol., vol. 8, pp. 335-375, 1997.

[3] L.-Y. Shao, X. Dong, A. P. Zhang, H.-Y. Tam, and S. He, "High-resolution strain and temperature sensor based on distributed Bragg reflector fiber laser," IEEE Photon. Technol. Lett., vol. 19, no. 20, pp. 1598-1600, Oct. 15, 2007.

[4] J. Capmany and Dalmanovak, "Microwave photonics combines two worlds," Nature Photon., vol. 1, pp. 319-330, 2007.

[5] Y. Yao, X. Chen, Y. Dai, and S. Xie, "Dual-wavelength erbium-doped fiber laser with a simple linear cavity and its application in microwave generation," IEEE Photon. Technol. Lett., vol. 18, no. 1, pp. 187-189, Jan. 1, 2006

[6] D. Chen, H. Fu, W. Liu, Y. Wei, and S. He, "Dual-wavelength single-longitudinal-mode erbium-doped fibre laser based on fibre Bragg grating pair and its application in microwave signal generation," Electron. Lett., vol. 44, pp. 459-461, 2008.

[7] R. N. Nogueira, I. Abe, A. J. Fernandes, H. J. Kalinowski, R. F. da Rocha, and J. L. Pinto, "Spatial characterization of fiber Bragg grating structures using transversal pressure," Opt. Commun., vol. 259, pp. 110-114, 2006.

[8] C. J. S. de Matos, P. Torres, L. C. G. Valente, W. Margulis, and R. Stubbe, "Fiber Bragg grating (FBG) characterization and shaping by local pressure," J. Lightw. Technol., vol. 19, no. 8, pp. 1206-1211, Aug. 2001.

[9] Z. C. Liu, Y. Xiufeng, L. Chao, N. J. Hong, G. Xin, P. R. Chaudhuri, and D. Xinyong, "Switchable multi-wavelength erbium-doped fiber lasers by using cascaded fiber Bragg gratings written in high birefringence fiber," Opt. Commun., vol. 230, pp. 313-317, 2004. 